\title{
Motion Compensation for SAA FMCW Radar Based on Specific Switching Scheme
}

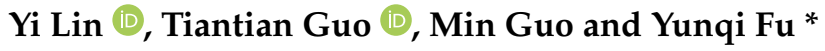 \\ College of Electronic Science and Technology, National University of Defense Technology, \\ Changsha 410073, China \\ * Correspondence: yunqifu@nudt.edu.cn; Tel.: +86-138-7499-2422
}

Received: 5 July 2019; Accepted: 16 August 2019; Published: 21 August 2019

\begin{abstract}
Switch antenna array (SAA) frequency modulated continuous wave (FMCW) radar has been studied by many researchers due to its low cost and straightforward architecture. However, the motion-induced phase leads to incorrect direction-of-arrival (DoA) estimation, which is one of the main obstacles when it is applied to a moving scenario. To address this problem, we propose a motion compensation method based on a specific switching scheme in this paper. The effectiveness of this method is verified by simulation and measurement results. This approach only requires basic signal processing techniques and avoids complex optimization algorithms.
\end{abstract}

Keywords: switch antenna array (SAA); frequency modulated continuous wave (FMCW) radar; motion compensation

\section{Introduction}

Switch antenna array (SAA) frequency modulated continuous wave (FMCW) radar has received extensive attention due to its low complexity architecture and small size. It has been widely used in scenes such as car collision avoidance and obstacle monitoring [1,2]. Figure 1 shows the system architecture of the SAA FMCW radar. The transmitting frontend uses a single antenna, and the receiving frontend adopts an N-elements uniform linear array (ULA), which shares a receiving channel through a single-pole multithrow switch.

In the conventional switching scheme, the receiving arrays are sequentially switched in order from 1 to $N$ [3]. For the stationary target, the phase difference between adjacent elements is expressed as

$$
\varphi_{d}=\beta d \sin \theta
$$

which is the same as the multichannel antenna array, where $\beta=2 \pi / \lambda$ is the wave propagation constant, $\lambda$ is the wavelength in free space, $d$ is the spacing of ULA, and $\theta$ is the direction-of-arrival (DoA). For the nonstationary target, the motion introduces an additional phase difference between adjacent elements, which is expressed as in the work by the authors of [4].

$$
\varphi_{v}=\beta 4 v T
$$

where $v$ is the target's velocity, $T$ is the up-chirp ramp duration, and the radar's pulse repetition interval (PRI) is $2 T$. The target moves with constant radial velocity and its tangential velocity can be neglected [5]. The phase distribution of SAA for the moving target is shown in Figure 2. 


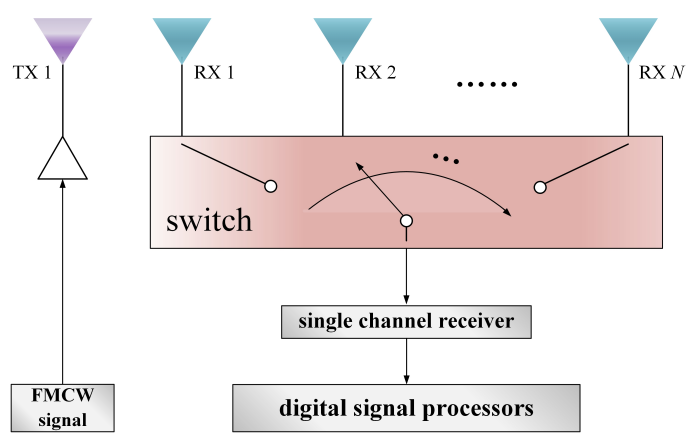

Figure 1. Block diagram of switch antenna array (SAA) frequency modulated continuous wave (FMCW) radar.

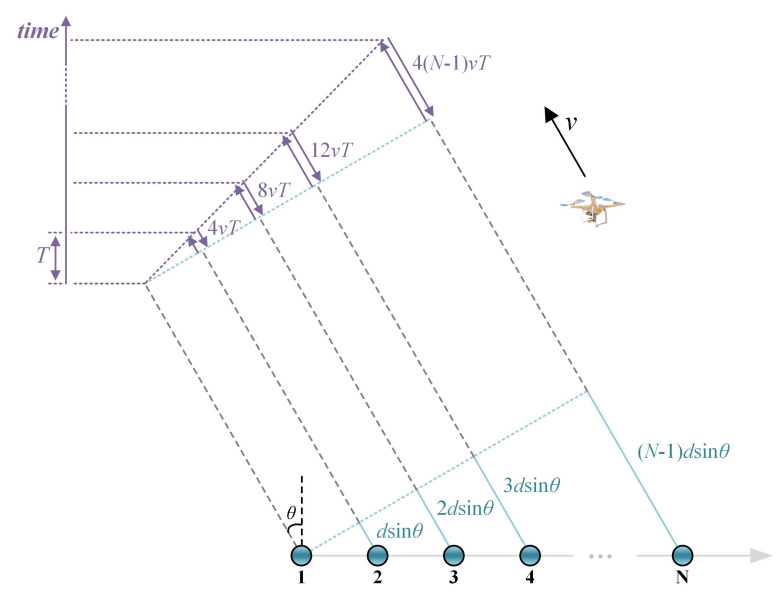

Figure 2. Phase distribution for moving target.

The motion-induced phase $\varphi_{v}$ will lead to erroneous DoA estimation [4]. Therefore, scholars have proposed some motion compensation methods to solve this problem. L. Yang proposed a two-cycle switching scheme to achieve DoA estimation [3]. However, this method is only applicable to scenes where the target moves at a slower speed. Some random switching methods were proposed in works by the authors of [5-7] to realize motion compensation. However, these methods need to combine the target scattering characteristics and optimization algorithms to solve the optimal switching scheme, consuming time and computing resources, making application to scenarios with high real-time requirements difficult. Yang [8] successfully estimated direction and velocity using two-dimensional MUSIC signal processing and M.-S. Lee [9] introduced the time-invariant steering vector (TISV) to achieve multiparameter (i.e., range, velocity, and direction) estimations. However, this approach suffers from an ambiguous velocity estimation problem. Lin [10] proposed a motion-induced phase elimination method based on double-time switching scheme. Some scholars have proposed compensation methods for time division multiplexing (TDM) multiple-input-multiple-output (MIMO) radars. Jonathan investigated a modified Doppler processing algorithm to achieve motion compensation [11]. The phase shift introduced by movement can be calculated using signals of redundant antenna elements [12,13]. Dominik and Ziroff [14] used the echo signal of the first antenna element to estimate a target's velocity, and then used this velocity to construct a term to compensate for the phase response of the moving target. Additionally, Guetlein [15] found that the phase difference introduced by the target motion can be ignored when the transmit antenna was quickly switched at a single frequency. However, these methods cannot be applied to SAA radars.

This paper proposes a specific switching scheme to compensate for the motion-induced phase for SAA FMCW radar. The receiving array is switched in the order of $1,4,2,3,5,6,7, \ldots, N$. 
The motion-induced phase is extracted through a simple mathematical operation, and then it is used to compensate the phase of SAA. The proposed method only requires basic signal processing techniques and avoids complex optimization algorithms.

\section{Motion-Induced Phase Compensation}

In this section, we describe the signal model of the SAA FMCW radar using a conventional switching scheme and proposed a specific switching scheme. Then, the principle and procedure of the motion compensation method based on the specific switching scheme are given.

For the conventional sequential switching scheme from 1 to $N$, the up-chirp beat signal received by the $n$th element is modeled similar to works by the authors of $[3,4]$ :

$$
\begin{aligned}
u_{n}(t) & =s(t) \exp \left[-j(n-1)\left(\varphi_{d}+\varphi_{v}\right)\right] \\
s(t) & =a \exp \left[j 2 \pi\left(f_{0} t+\phi_{0}\right)\right]
\end{aligned}
$$

where $s(t)$ is the beat signal received by the 1st element, $a$ is signal amplitude, $f_{0}=2 k r / c+2 v / \lambda$ is frequency information, $\phi_{0}=2 r / \lambda$ is phase information, $k$ is the chirp rate, $c$ is the light speed constant, and $r$ is the target's range. Note that the triangular wave consisting of up-chirp and down-chirp is used in this paper.

The motion-induced phase $\varphi_{v}$ leads to erroneous DoA estimation [4], and the incorrect DoA $\theta_{e}$ is determined by the following formula.

$$
\sin \theta_{e}=\sin \theta+\frac{2 m \pi-\varphi_{v}}{\beta d} ; m=0, \pm 1, \pm 2, \ldots
$$

To compensate for the motion-induced phase and estimate DoA correctly, this paper proposes a specific switching scheme as shown in Figure 3. The receiving elements are switched in the order of $1,4,2,3,5,6,7, \ldots, N$, which is different from the conventional sequential switching scheme from 1 to $N$.

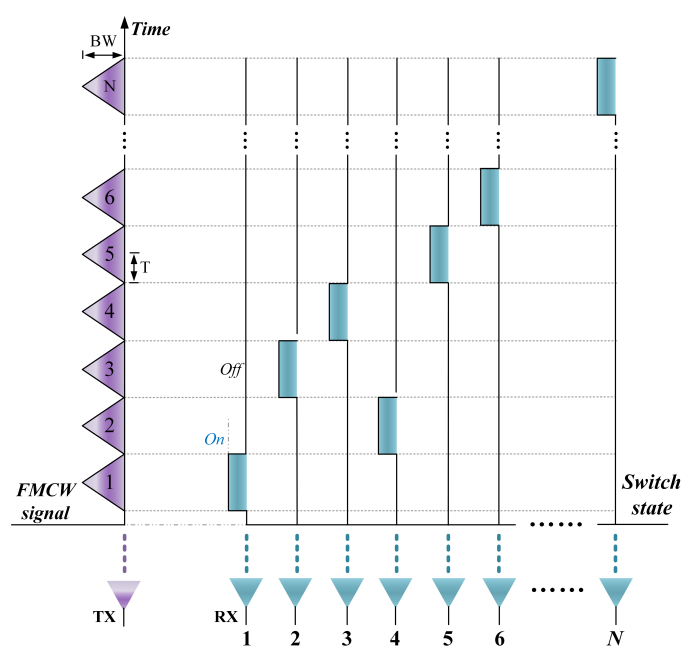

Figure 3. Timing diagram of specific switching scheme. 
Figure 4 illustrates the signal processing flow of the proposed motion compensation method. The receiving elements are switched according to the specific switching scheme to obtain a raw array signal, which is expressed as

$$
\begin{array}{ll}
s_{1}(t)=s(t) ; & n=1 \\
s_{2}(t)=s(t) \exp \left[-j\left(\varphi_{d}+2 \varphi_{v}\right)\right] ; & n=2 \\
s_{3}(t)=s(t) \exp \left[-j\left(2 \varphi_{d}+3 \varphi_{v}\right)\right] ; & n=3 \\
s_{4}(t)=s(t) \exp \left[-j\left(3 \varphi_{d}+\varphi_{v}\right)\right] ; & n=4 \\
s_{n}(t)=s(t) \exp \left[-j\left((n-1) \varphi_{d}+\varphi_{v}\right)\right] ; & n=5,6, \ldots, N
\end{array}
$$

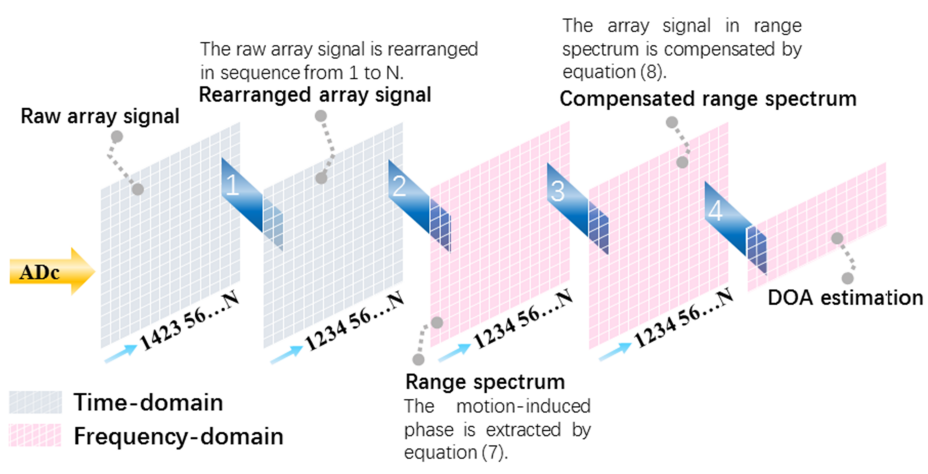

Figure 4. Signal processing flowchart for motion compensation.

A fast Fourier-transform (FFT) leads to the range spectrum

$$
\begin{array}{ll}
x_{1}\left(r^{e s}\right)=b\left(r^{e s}\right) \exp \left(-j \varphi_{0}\right) ; & n=1 \\
x_{2}\left(r^{e s}\right)=b\left(r^{e s}\right) \exp \left[-j\left(\varphi_{d}+2 \varphi_{v}+\varphi_{0}\right)\right] ; & n=2 \\
x_{3}\left(r^{e s}\right)=b\left(r^{e s}\right) \exp \left[-j\left(2 \varphi_{d}+3 \varphi_{v}+\varphi_{0}\right)\right] ; & n=3 \\
x_{4}\left(r^{e s}\right)=b\left(r^{e s}\right) \exp \left[-j\left(3 \varphi_{d}+\varphi_{v}+\varphi_{0}\right)\right] ; & n=4 \\
x_{n}\left(r^{e s}\right)=b\left(r^{e s}\right) \exp \left[-j\left((n-1)\left(\varphi_{d}+\varphi_{v}\right)+\varphi_{0}\right)\right] ; & n=5,6, \ldots, N
\end{array}
$$

where $r^{e s}$ is the estimated range of the target; $b\left(r^{e s}\right)$ and $\varphi_{0}$ are the amplitude and phase information, respectively. Targets with close DoAs are differentiated by the range cell in the range spectrum. The phase compensation method in this paper is to perform phase extraction and phase compensation for each range cell independently. Therefore, the proposed method is also be efficient for these targets.

Observing the phase information of the first three elements, we found that the phase information of the motion-induced phase $\varphi_{v}$ and the elements satisfies the following formula.

$$
\varphi_{v}=2 \psi_{2}-\psi_{3}-\psi_{1}
$$

where $\psi_{1}=\varphi_{0}, \psi_{2}=\varphi_{d}+2 \varphi_{v}+\varphi_{0}, \psi_{3}=2 \varphi_{d}+3 \varphi_{v}+\varphi_{0}$ are the phase information of the 1st, 2nd, and 3rd elements, respectively.

Assuming the extracted motion-induced phase is $\widehat{\varphi}_{v}$, the compensated range spectrum is denoted by

$$
\begin{aligned}
& \hat{x}_{1}\left(r^{e s}\right)=x_{1}\left(r^{e s}\right) ; \quad n=1 \\
& \hat{x}_{2}\left(r^{e s}\right)=x_{2}\left(r^{e s}\right) \exp \left(-j 2 \widehat{\varphi}_{v}\right) ; \quad n=2 \\
& \widehat{x}_{3}\left(r^{e s}\right)=x_{3}\left(r^{e s}\right) \exp \left(-j 3 \widehat{\varphi}_{v}\right) ; \quad n=3 \\
& \hat{x}_{4}\left(r^{e s}\right)=x_{4}\left(r^{e s}\right) \exp \left(-j \widehat{\varphi}_{v}\right) ; \quad n=4 \\
& \hat{x}_{n}\left(r^{e s}\right)=x_{n}\left(r^{e s}\right) \exp \left[-j(n-1) \widehat{\varphi}_{v}\right] ; \quad n=5,6, \ldots, N
\end{aligned}
$$


The compensated range spectrum can be directly applied to estimate DoA. Note that the raw array signal is rearranged in the order of 1 to $N$ for DoA estimation.

\section{Simulation and Measurement}

Table 1 shows the numerical and experimental radar parameters. The Bartlett beamformer with $30 \mathrm{~dB}$ Chebyshev attenuation is used for DoA estimation in this paper.

The simulation was conducted without noise to avoid uncertainty. Two point scatterings with identical amplitude were considered as moving targets. Their ranges, velocities, and azimuths were $\left\{r_{1}, v_{1}, \theta_{1}\right\}=\left\{30 \mathrm{~m},-100 \mathrm{~m} / \mathrm{s}, 0^{\circ}\right\}$ and $\left\{r_{2}, v_{2}, \theta_{2}\right\}=\left\{40 \mathrm{~m}, 50 \mathrm{~m} / \mathrm{s},-30^{\circ}\right\}$. Figure 5 shows the phase characteristic curve of SAA with and without compensation. There was significant distortion in the uncompensated phase characteristic due to the motion-induced phase. In contrast, the compensated phase characteristic was in good agreement with the theoretical value, which represents the phase characteristic of the multichannel antenna array. Figure 6 shows the DoA estimation results with and without compensation. The DoA estimations without compensation were $-15^{\circ}$ and $-21^{\circ}$, respectively, deviating from the true angles of the targets. The fluctuation of the side lobes was caused by the nonlinear phase of the first four elements. With compensation, the DoA estimations were $1^{\circ}$ and $-31.5^{\circ}$, which were close with the actual values. Meanwhile, the side-lobe level was reduced overall. The measurement error is caused by the high-order term of the beat signal. For the received signal of a single element, the phase error introduced by the high-order term can be neglected [16], so this paper and works of the authors of [3,4] use the simplified signal model represented by Equation (3) for phase analysis. However, in this paper, the received signals of the first three units are used to extract the phase of motion introduction, so that the high-order term causes measurement error.

Table 1. Radar parameters for simulation and measurement.

\begin{tabular}{lll}
\hline & Simulation & Measurement \\
\hline Operating frequency: $f$ & $10 \mathrm{GHz}$ & $10.25 \mathrm{GHz}$ \\
Modulation period: $T$ & $10 \mu \mathrm{s}$ & $0.1 \mathrm{~ms}$ \\
Sweep bandwidth: $B W$ & $100 \mathrm{MHz}$ & $100 \mathrm{MHz}$ \\
Number of elements: $N$ & 32 & 8 \\
Spacing: $d_{r}$ & $0.5 \lambda$ & $0.52 \lambda$ \\
Type of element & Ideal element with cosine pattern & SIW slot array \\
\hline
\end{tabular}

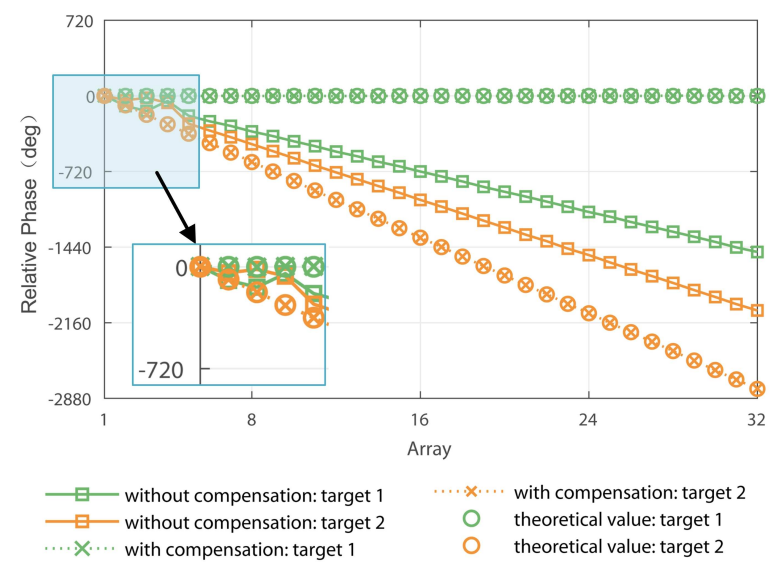

Figure 5. Phase characteristic curve of SAA. 


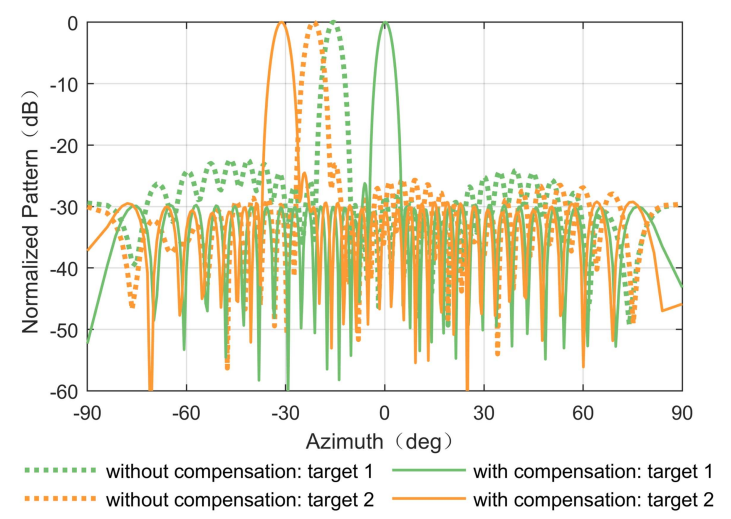

Figure 6. Direction-of-arrival (DoA) estimation results.

Figure 7 shows the experimental scenario. The target is the unmanned aerial vehicle (UAV): DJI Phantom 3. It rose vertically at a speed of $3 \mathrm{~m} / \mathrm{s}$ at a distance of $80 \mathrm{~m}$ from the radar. The antenna was placed parallel to the ground to avoid ground clutter. Figure 8 shows the phase characteristic curve of SAA with and without compensation. Without compensation, the phase characteristic had motion-induced distortion. With compensation, the phase characteristic was consistent with the theoretical value. Figure 9 shows the DoA estimation results with and without compensation. Without compensation, the DoA was incorrectly estimated at $-4.3^{\circ}$. With compensation, the DoA was correctly estimated at $0^{\circ}$. Note that the side-lobe level deteriorated due to the ambient noise. Additionally, we also used the TISV-based multiparameter estimation algorithm [8] to estimate DoA and obtained the real DoA of UAV at $0^{\circ}$.

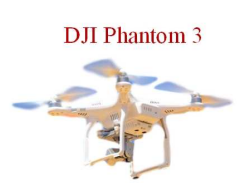

(a)

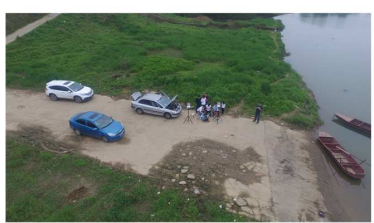

(b)

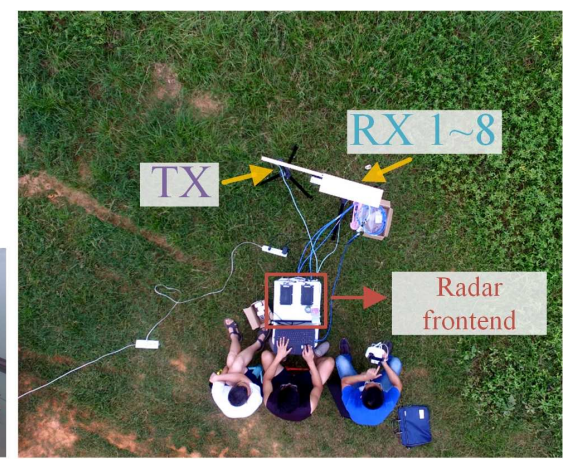

(c)

Figure 7. Measurement scenario: (a) unmanned aerial vehicle (UAV) as target, (b) experimental environment, and (c) top view taken by UAV.

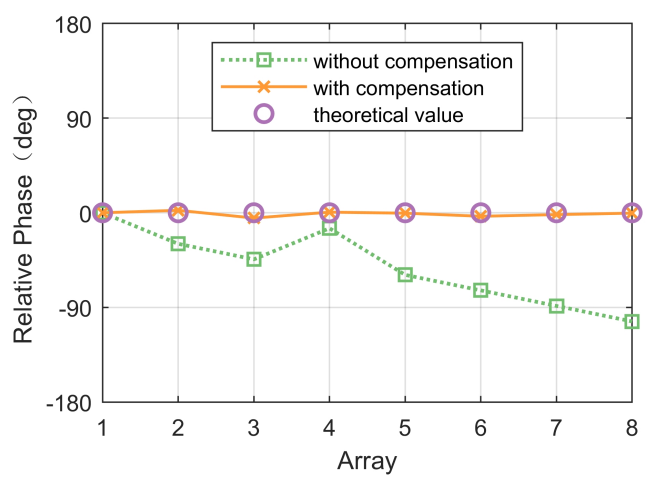

Figure 8. Phase characteristic curve of SAA. 


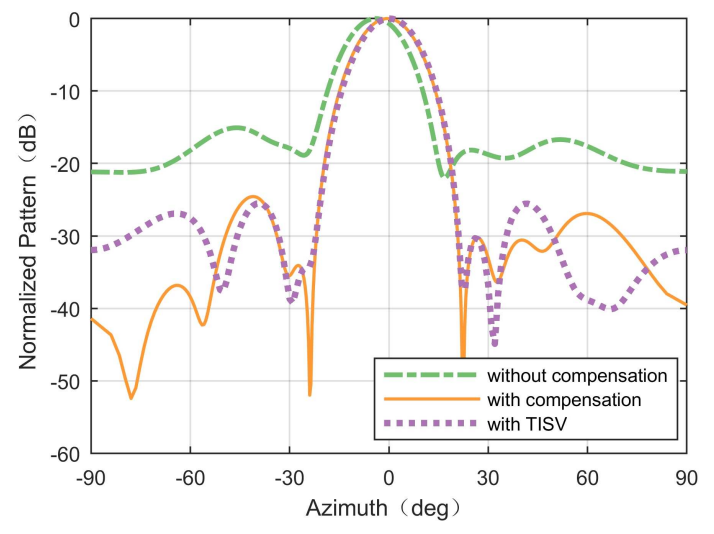

Figure 9. DoA estimation results.

\section{Conclusions}

This letter introduces a specific switching scheme to realize a motion compensation method for SAA FMCW radar. The receiving array is switched in the order of $1,4,2,3,5,6,7, \ldots, N$. The motion-induced phase is calculated, and then it is used to complete motion compensation. The simulation and measurement results demonstrate the performance of the proposed motion compensation method. Compared to previously published methods for motion compensation, the proposed method only requires basic signal processing techniques and avoids complex optimization algorithms. In the future, we will integrate conventional radar signal processing techniques, including moving target indicator (MTI), moving target detector (MTD), and constant false alarm rate (CFAR) detection, to achieve more abundant functions, such as multiparameter estimations, target detection and tracking, etc.

Author Contributions: Y.L. and Y.F. wrote the paper; Y.F. provided the idea and instruction for this paper. T.G. revised this paper; Y.L. and M.G. implemented and analyzed the simulation. All authors contributed to this manuscript.

Funding: This research was supported by the National Natural Science Foundation of China under Grant No. 61571448.

Conflicts of Interest: The authors declare no conflicts of interest.

\section{References}

1. Lee, S.; Joo, J.; Choi, J.; Kim, W.; Kwon, H.; Lee, S.; Kwon, Y.; Jeong, J. W-band multichannel FMCW radar sensor with switching-TX antennas. IEEE Sens. J. 2016, 16, 5572-5582. [CrossRef]

2. Ganis, A.; Navarro, E.M.; Schoenlinner, B.; Prechtel, U.; Meusling, A.; Heller, C.; Spreng, T.; Mietzner, J.; Krimmer, C.; Haeberle, B.; et al. A portable 3-D imaging FMCW MIMO Radar Demonstrator with a $24 \times 24$ antenna array for medium-range applications. IEEE Trans. Geosci. Remote Sens. 2018, 56, 298-312. [CrossRef]

3. Yang, L.; Zhenghe, F. Switch antenna array with single receiving channel for FMCW radar. In Proceedings of the International Symposium on Antennas and Propagation, Fukuoka, Japan, 21-25 August 2000; Volume 4, pp. 1629-1632.

4. Lin, Y.; Yang, K.; Guo, M.; Fu, Y. Direction estimation of low-SNR moving targets for SAA FMCW radar. IEEE Access 2018, 6, 69371-69380. [CrossRef]

5. Hu, C.; Liu, Y.; Meng, H.; Wang, X. Randomized switched antenna array FMCW radar for automotive applications. IEEE Trans. Veh. Technol. 2014, 63, 3624-3641. [CrossRef]

6. Belfiori, F.; Van Rossum, W.; Hoogeboom, P. Random transmission scheme approach for a FMCW TDMA coherent MIMO radar. In Proceedings of the 2012 IEEE Radar Conference, Atlanta, GA, USA, 7-11 May 2012; pp. 178-183.

7. Hu, X.; Lu, M.; Li, Y.; Wang, Y. Motion compensation for TDM MIMO radar by sparse reconstruction. Electr. Lett. 2017, 53, 1604-1606. [CrossRef] 
8. Yang, L.; Liwan, L.; Weifeng, P.; Yaqin, C.; Zhenghe, F. Signal processing method for switch antenna array of the FMCW radar. In Proceedings of the IEEE National Radar Conference, Atlanta, GA, USA, 3 May 2001; pp. 289-293.

9. Lee, M.S.; Katkovnik, V.; Kim, Y.H. System modeling and signal processing for a switch antenna array radar. IEEE Trans. Signal Process. 2004, 52, 1513-1523. [CrossRef]

10. Lin, Y.; Yang, K.; Guo, M.; Fu, Y. Elimination of motion-induced phase based on double-time switching scheme for SAA FMCW radar. Electronics 2019, 8, 786. [CrossRef]

11. Bechter, J.; Roos, F.; Waldschmidt, C. Compensation of motion-induced phase errors in TDM MIMO Radars. IEEE Microw. Wirel. Compon. Lett. 2017, 27, 1164-1166. [CrossRef]

12. Schmid, C.M.; Feger, R.; Pfeffer, C.; Stelzer, A. Motion compensation and efficient array design for TDMA FMCW MIMO radar systems. In Proceedings of the 6th European Conference on Antennas and Propagation, EuCAP 2012, Prague, Czech Republic, 26-30 March 2012; pp. 1746-1750.

13. Feger, R.; Wagner, C.; Schuster, S.; Scheiblhofer, S.; Jager, H.; Stelzer, A. A 77-GHz FMCW MIMO radar based on an SiGe single-chip transceiver. IEEE Trans. Microw. Theory Tech. 2009, 57, 1020-1035. [CrossRef]

14. Dominik, Z.; Ziroff, A. Phase migration effects in moving target localization using switched MIMO arrays. In Proceedings of the 2015 European Radar Conference, Paris, France, 9-11 September 2015; pp. 85-88.

15. Guetlein, J.; Bertl, S.; Kirschner, A.; Detlefsen, J. Switching scheme for a FMCW-MIMO radar on a moving platform. In Proceedings of the European Microwave Week 2012: “Space for Microwaves", EuMW 2012, 9th European Radar Conference, EuRAD 2012, Amsterdam, The Netherlands, 31 October-2 November 2012; pp. 91-94.

16. Stove, A. Linear FMCW radar techniques. IEE Proc. Part F Radar Signal Process. 1992, 139, 343-350. [CrossRef]

(C) 2019 by the authors. Licensee MDPI, Basel, Switzerland. This article is an open access article distributed under the terms and conditions of the Creative Commons Attribution (CC BY) license (http:/ / creativecommons.org/licenses/by/4.0/). 